\title{
High working memory performers have efficient eye movement control systems under Reading Span Test
}

\author{
Miyuki Azuma \\ Osaka University
}

Takashi Ikeda

Osaka University

Mariko Osaka

Osaka University

\author{
Takehiro Minamoto \\ Kyoto University \\ Naoyuki Osaka \\ Kyoto University
}

\begin{abstract}
Controlled eye movements are critical in performing highly goal oriented behavior such as text reading. Previous studies have examined the relationship between working memory capacity and eye movement control during working memory task. However, the results were inconsistent, due to a methodological issue including the predictability of target location. In the present study, we used Japanese version of reading span test, where the position of to-be-remembered word is not predictable so that more efficient attentional control is required, and investigated how working memory capacity contributes to eye movement control during reading span test. Results based on total fixation time revealed that highworking memory performers efficiently control or shift their attention under high memory load.
\end{abstract}

Keywords: Working memory, Reading Span Test, individual difference,

Eye movement research during reading has a long history dating back to the late 19th century, and several models of eye movement control in reading have been proposed (Rayner, 2008; Vitu, 2008). In our everyday life, we rely on visual attentional control for goal-oriented activities like reading. The goal of reading is to understand the meaning of text with precisely-controlled eye movements that provide enough fixation time to read the text (Osaka \& Osaka, 1994). Moreover, an individual utilizes attentional control in the brain for information manipulation and storage using verbal working memory to understand what he or she has just read. Working memory supports immediate brain processes involved in the storage and processing of information and is considered to play a particularly critical role in comprehension processes during text reading. (Daneman \& Carpenter, 1980). For example, incoming text information is decoded perceptually, recognized, and stored for short periods of time in working memory while being integrated into a sentence and interpreted. During this process, working memory plays an important function in the stor- age of intermediate or final products, allowing readers to integrate the contents of text into context using eye movement control (Just \& Carpenter, 1992).

Verbal working memory plays an important role in text reading and language comprehension. According to Baddeley (1986), the working memory system supports the simultaneous storage and processing of information. In reading comprehension, the system is assumed to store information or the product of preceding text and use it to process and integrate subsequent text.

One of the most frequently-employed method for measuring verbal working memory capacity is the Reading Span Test (RST). In the RST, participants are asked to read a series of short sentences aloud and simultaneously memorize the last word of each sentence. Then, participants are asked to perform a dual task (i.e., reading aloud /understanding a sentence and memorizing the last word of each sentence). As the number of sentences increases from one to five, participants are required to maintain up to five words for a subsequent recall test. Scores on the RST have been found to be highly correlated with reading comprehension; however, reading 
Journal of Eye Movement Research

$5(3): 1,1-10$

comprehension scores have been found to be less correlated with scores of short-term memory (Daneman \& Carpenter, 1980; Daneman \& Merikle, 1996).

Osaka \& Osaka (1994) developed the RST in Japanese to compare to the English version of the RST developed by Daneman \& Carpenter (1980), and to examine the test's correlation with other criteria. As a result, the Japanese-RST, like the English-RST, has been shown to be a reliable measure of verbal working memory. It should be noted that the Japanese RST differs from the English RST on the following points: 1) the position of the target word; and 2) the role of the target word in text. In the English RST, the position of a target word is always the last word in the sentence. In the Japanese RST, the position of the target word is changed in a given trial and a target word is highlighted by a thin red underline (Osaka \& Osaka, 1994). The Japanese RST is comparable in validity to different versions of the RST, such as those in English, German, and French, and is considered to be a measure of verbal working memory capacity that is independent of language (Osaka \& Osaka, 1992; Osaka, Osaka \& Groner, 1993).

\section{Previous studies of eye movement using RST}

Carpenter \& Just (1989) investigated the relationship between working memory capacity and eye movement using the RST. In the study, a word in a sentence disappeared as soon as a participant had read it in order to prevent re-reading, and eye fixation on the to-beremembered word was analyzed. The result showed that the individuals with high spans could read a sentence faster and spent more time on the to-be-remembered words, relative to the individuals with lower span.

Engle, Cantor, \& Carullo (1992) showed a result consistent with Carpenter and Just (1989). They used an English version of the RST in which the to-be-remembered word (target word) was separately presented from a sentence and semantically unrelated to the content of the sentence. In the test, they prepared two reading conditions:1) the read only condition (i.e., sentence-word task without recall); and 2) the read and recall condition (i.e., sentence-word task). Under both conditions, participants read a sentence and the following word aloud. During the task, a semantic judgment task was given at a random interval and participants were required to judge whether the sentence was semantically correct or not. In the read
Azuma, M., Ikeda, T., Minamoto, T., Osaka, M., \& Osaka, N. (2012) Working memory and eye movement control under Reading Span Test

and recall condition, participants were further required to remember the target word in a sentence-word string. Set size was manipulated from two to six. The task was subject-paced, so participants could control their reading time. Analysis of the viewing time showed that, in the read and recall condition, the high-span group spent a longer time on the first and last word of sentence as well as the to-be-remembered word relative to the low-span group. These results indicate that high span participants direct their attention toward goal-relevant information more efficiently than low span participants.

Kaakinen \& Hyönä (2007) used a Finnish version of the original reading span test in which to-be-remembered word (i.e., target word) was the last word of the sentence. The task was experimenter-paced, where the experimenter terminated each sentence presentation as soon as participants finished vocalizing the sentence, and investigated how working memory capacity affects eye movement during sentence reading. They compared the eye movement among the three span groups under RST task of four-sentence condition. The investigators found that participants spent less time on the first and middle words of sentences and a longer time on the target word as memory load increased; however, fixation time did not differ among the groups.

The discrepancy in prior results may be attributed to differences in methodology; other studies used different version of RST, and the task was subject-paced in one study and experimenter-paced in another study. In the Kaakinen \& Hyönä (2007), study target words were always placed at the end of sentences, which enabled the participants to predict where to allocate their attention. That procedure might remove the effect of working memory capacity on the eye movement control. On the other hand, in the study of Engel et al. (1992), participants were allowed to perform the task in their own pace (subject-paced). In that situation, high working memory performers might control the viewing time by staying at a sentence for a longer period to memorize the words. Such a viewing strategy might contribute to the superior performance of high working memory individuals compared to the low working memory individuals. In the study of Carpenter \& Just (1989), low span participants might have difficulty to adjust to the experimental situation. Specifically, removal of viewed words might capture their attention, preventing them from focusing on the target words. Considering those problems in the previous 
studies, the present study made two modifications in the reading span test. The first modification is that we assigned target words at an unpredictable location. This procedure is expected to demand more precise eye movement control, which would accentuate individual differences in eye movement control between high- and low-span individuals. The second modification is that we employed an experimenter-paced RST, in which an experimenter strictly controlled the viewing time. This procedure will remove the effect of a viewing strategy. By applying the two modifications to the Japanese version of RST, we examined whether individual differences in working memory capacity is related to efficiency of eye movement control.

We were also interested in how eye movement during the RST differs from that of normal sentence reading in terms of attentional control. In order to make sure that the RST requires a different way of reading from traditional reading, we compared eye movements while participants only read a sentence versus when participants read the sentence and remembered the word simultaneously. In the study of Engle et al. (1992), the sentence-word task without recall could be considered as a dual task since participants needed to store the contents of sentences for semantic judgments. In the present study, we excluded any storage components in the usual reading condition so that participants read every sentence under no memory load.

In addition to eye movement, we measured reading time of task sentence, and compared the time of the high span group with that of the low span group. As mentioned above, Carpenter \& Just (1989) showed that high span individuals showed faster sentence reading than low span individuals while high span individuals spent longer time on the to-be-remembered words. In the study of Friedman and Miyake (2004), they compared reading time of high span individuals with that of low span individuals, manipulating memory load. As the result, they found that low span individuals took longer time under high memory load. Considering those findings, it is predicted that reading time would differ between high and low span groups under higher memory load.

\section{Method}

\section{Participants}

Participants were undergraduate and graduate students from Osaka University ( $n=51$, age range 19 - 32). Twenty-one participants were removed from data analysis because of technical issues; malfunction of the eyetracking system $(n=15)$, extreme head movements $(n=$ $2)$, or repetitive failure of calibration $(n=4)$. Remaining participants were divided into two groups (i.e., high- and low-performers) based on their RST score, which was measured prior to the experiment with the Japanese version of the RST (Osaka et al. 1994). The high-span group was comprised of 14 participants, and the low-span group was comprised of 16 participants. The mean span of the high-span group (HS) and the low-span group (LS) was $4.21(3.5-5.0)$ and $2.56(2.0-3.0)$, respectively. All participants gave their informed consent in accordance with the Department of Human Sciences, Osaka University.

\section{Eye movement recording}

Eye movements were recorded with a Tobii T120 eye tracker (Tobii Technology AB, Danderyd, Sweden) using a $120 \mathrm{~Hz}$ data sampling rate and an automatic calibration procedure with 5 calibration points: one center and four corners. The maximum error in the calibration was 30 pixels in diameter. A chin rest was used to restrict participant's head movements with a viewing distance of 57 $\mathrm{cm}$. As the eye tracking system had a large freedom of head movement $(30 \times 22 \times 30 \mathrm{~cm})$, we could collect good-quality data under oral reading.

\section{Materials}

For the eye movement experiment, we employed another version of the RST, as participants had experienced the standard RST (Osaka et al., 1994) to measure their working memory span. All sentences consisted of Japanese Kana and Kanji. Mean sentence lengths were 24.7 characters and 30.7 moras. The target words were all nouns consisting of 1 to $3 \mathrm{Kanji}$ characters. The size of a letter was 34 pixels, extending a visual angle of 0.9 degrees. The visual angle of each sentence was approximately 23 degrees. 
Journal of Eye Movement Research

$5(3): 1,1-10$

\section{Procedure}

There were two conditions in the experiment, namely, the RST condition and the READ condition. In the RST condition, participants were asked to read a sentence and memorize the target word simultaneously. In the READ condition, participants were asked to read a sentence orally. We introduced the two-sentence (low memory load) and five-sentence condition (high memory load). Each condition consisted of three trials. One example sentence is Much of his memory regarding the war is unclear, and its target word is memory. Figure 1 shows an example of the two-sentence condition.

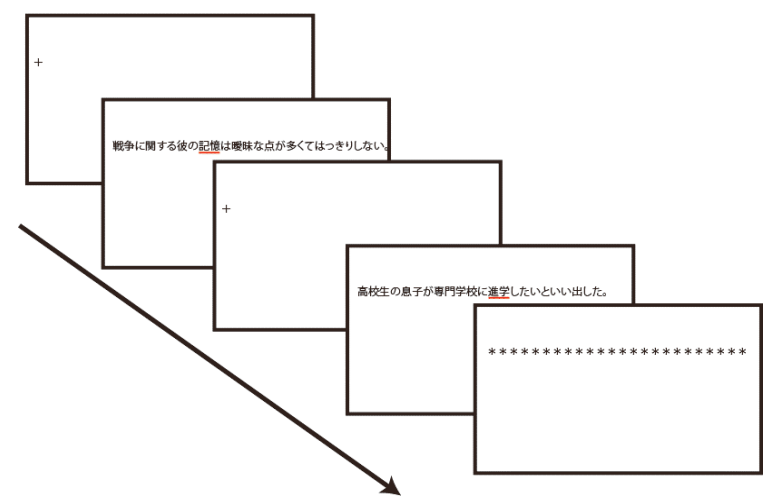

Figure 1. Example of presentation sequence for the twosentence task. (English translation of the first sentence; Much of his memory regarding the war is mostly unclear, and its target word is memory, the second sentence; My son is high school student and claims to go on to technical college, and its target word is shingaku, a noun that means to go to a higher school) Participants are instructed to remember the underlined word under the RST condition but are not required to remember the word under the READ condition.

Before each sentence was presented, participants were instructed to gaze at a fixation point presented for $500 \mathrm{msec}$. The fixation point was replaced by the first letter of a sentence. Participants were required to read each sentence aloud as soon as it was presented. We employed the experimenter-paced method, following the procedure of Kaakinen \& Hyönä's study (2007). When participants finished reading the sentence aloud, the presentation of the sentence was terminated by an experimenter and the next sentence immediately appeared. After all sentences were presented, participants recalled target words orally in the RST condition and counted the number of presented sentences in the READ condition. The order of conditions and sentences included in each condition were quasi-randomized. In the present study, a to-be-remembered word and a cue underline was pre-
Azuma, M., Ikeda, T., Minamoto, T., Osaka, M., \& Osaka, N. (2012) Working memory and eye movement control under Reading Span Test

sented simultaneously, and the cue remained on a monitor until the end of a trial.

\section{Data Analysis}

Data were analyzed using Tobii Studio 2.2.8. When the eye remained more than $50 \mathrm{~ms}$, we defined it as a fixation using an automatic fixation detection algorithm (Tobii Fixation Filter, detection radius of 17 pixels on a screen with a resolution of $1280 \times 1024$ pixels). We included all fixation data for the statistical analysis. The present study analyzed total fixation time in order to compare it with the results of the previous studies (Engle et al., 1992; Kaakinen \& Hyönä, 2007).

\section{Result}

We report the result as following order: (1) RST score, (2) Reading time of whole sentence, and (3) Total fixation duration including all the fixations on the target word.

\section{RST Score}

In the two sentence condition, the mean number of correctly recalled words was 5.6 out of 6 words for the HS $(S D=0.49)$, and 5.5 for LS $(S D=0.51)$. Thus, both the HS and the LS could recall correct words almost perfectly in the two-sentence condition. In fact, two groups did not differ in the score statistically, $F(1,28)=0.59, p$ $>.05$. In contrast, the mean number of correctly recalled words in the five-sentence condition was 11.5 words out of 15 words in the HS $(S D=1.69)$ and 7.25 words out of 15 words in LS $(S D=3.33)$. In the five-sentence condition, the mean span score was significantly higher in the HS relative to that of the $\operatorname{LS}, F(1,28)=18.46, p<.01$.

\section{Reading Time}

\section{Span group, task difficulty, and reading condition}

Figure 2 shows the whole sentence reading time of high-and low-span groups under four conditions.

In the low memory load condition, or two-sentence condition, the mean sentence reading time in the RST condition was $5,253 \mathrm{msec}(S D=636)$ for $\mathrm{HS}$, and $4,861 \mathrm{msec}(S D=.549)$ for LS. In the READ condition, it took $4,863 \mathrm{msec}(S D=581)$ for $\mathrm{HS}$, and $4,612 \mathrm{msec}$ $(S D=498)$ for LS. 


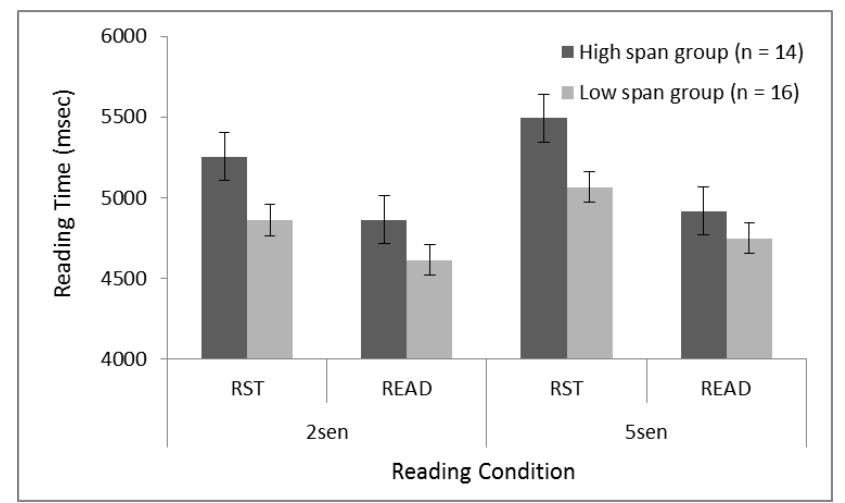

Figure 2. Reading time for the high-span group and low-span group for READ and RST under the easy 2 sentence condition and difficult 5 sentence condition.

In the high memory load condition, or five-sentence condition, the mean sentence reading time in the RST condition was 5,493 $\mathrm{msec}(S D=803)$ for HS, and 5,064 msec $(S D=638$ ) for LS. In the READ condition, it took $4,917 \mathrm{msec}(S D=.507)$ for $\mathrm{HS}$, and $4,748 \mathrm{msec}(S D$ $=.545)$ for LS.

A mixed 2 (Span group: HS, LS) $\times 2$ (Reading condition: RST, READ) × 2 (Task difficulty: 2-sentence, 5sentence) analysis of variance (ANOVA) was conducted. In this analysis, span group was a between-subject factor, whereas reading condition and task difficulty were within-subject factors. Result showed a main effect of the reading condition, $F(1,28)=57.16, p<.001$, indicating that the participants needed more time in the RST condition than the READ condition. A main effect of the task difficulty was also significant, $F(1,28)=20.02, p<.01$, which indicates that greater number of sentences required longer reading time. The main effects of span group was not significant, $F(1,28)=2.24, p>.05$. None of two-way interactions were significant; $F(1,28)=3.91, p>.05$ for the span group $\mathrm{x}$ reading condition, $F(1,28)=2.8, p$ $>.05$ for the task difficulty $\mathrm{x}$ reading condition, and $F$ (1, $18)=0.9, p>.05$ for the span group $\mathrm{x}$ task difficulty. Three-way interaction was not obtained, neither, $F(1,28)$ $=0.6, p>.05$. In sum, reading condition and task difficulty affected reading time, but not individual differences in working memory capacity.
Azuma, M., Ikeda, T., Minamoto, T., Osaka, M., \& Osaka, N. (2012 Working memory and eye movement control under Reading Span Test

\section{Total Fixation time}

\section{Span group, task difficulty, and reading condition}

Figure 3 and 4 shows the total fixation time on the target words in low (2 sentences) and high memory load (5 sentences) condition.

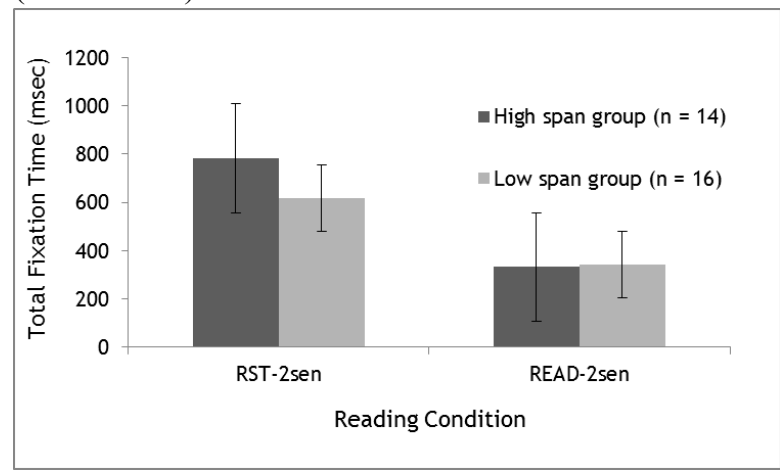

Figure 3. Total fixation time (s) for the high-span group and low-span group for READ and RST under the two-sentence condition.

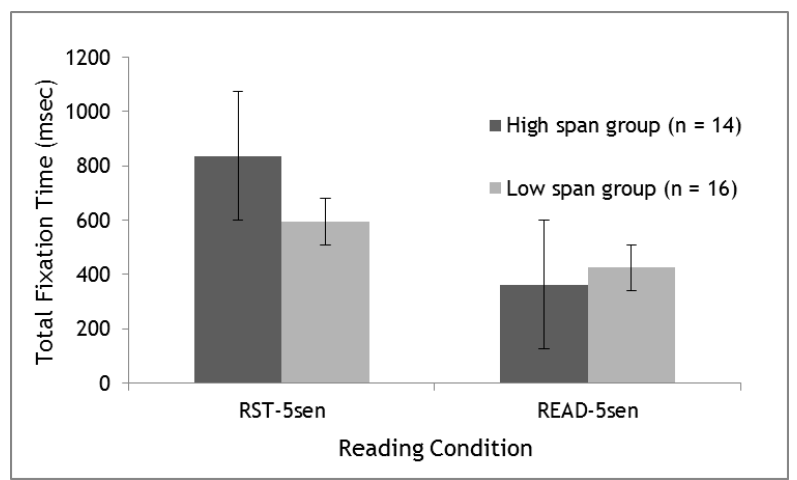

Figure 4. Total fixation time (s) for the high-span group and low-span group for READ and RST under the five-sentence condition.

In both the RST and READ conditions, total fixation time was computed for the HS and LS groups under both two- and five-sentence conditions.

In the low memory load condition, or two-sentence condition, the total fixation time on TBR word (i.e., tobe-remembered word) in the RST condition was 783 $\operatorname{msec}(S D=442)$ for HS, and $618 \mathrm{msec} \quad(S D=213)$ for LS. As for the READ condition, total fixation time on target word was $332 \mathrm{msec}(S D=193)$ for HS, and 342 msec $(S D=117)$ for LS (see Figure 3$)$, although participants were not required to remember the word. In the 
Journal of Eye Movement Research

$5(3): 1,1-10$

high memory load condition, total fixation time on TBR word in the RST condition was $836 \mathrm{msec}(S D=389)$ for $\mathrm{HS}$, and $594 \mathrm{msec}(S D=225)$ for LS. As for the READ condition, total fixation time on target word took 362 $\operatorname{msec}(S D=174)$ for HS, and $425 \mathrm{msec}(S D=139)$ for LS.

A mixed 2 (Span group: HS, LS) $\times 2$ (Reading condition: RST, READ) × 2 (Task difficulty: 2-sentence, 5sentence) analysis of variance (ANOVA) was conducted. As with the reading time analysis, span group was a between-subject factor, whereas reading condition and task difficulty were within-subject factors. Result showed a main effect of reading condition, $F(1,28)=65.86, p$ $<.001$, indicating that target words were fixated more in the RST condition in comparison to the READ condition. The main effect of task difficulty was also significant, $F$ $(1,28)=4.43, p<.05$, suggesting that the target word was more fixated in the high-load condition than the lowload condition. A main effect of the span group was not significant, $F(1,28)=1.10, p>.05$. Critically, there was a significant two-way interaction between the span group and the reading condition, $F(1,28)=8.11, p<.01$. Other two-way interactions was non-significant, $F(1,28)=$ $0.96, p>.05$ for the interaction between the reading condition and the task difficulty, and $F(1,28)=.12, p>.05$ for that between the span group and the task difficulty. Three-way interaction did not reach significant level, $F$ (1, $28)=2.35, p>.05$. As we found a significant interaction between the span group and reading condition, we separately examined the interaction in the 2-sentence condition and the 5-sentence condition. This analysis allowed us to test whether the factor of the span group and that of the reading condition similarly interacts across memory load. As the previous studies have shown that individual differences in working memory capacity (WMC) are accentuated as efficient attentional control is required (e.g., Kane and Engle, 2003), we hypothesized that a factor of the WMC interacts with reading conditions in the 5sentences condition.

\section{Two-sentence condition}

A $2 \times 2$ ANOVA with span group (HS and LS) and reading condition (RST and READ condition) showed a main effect for reading condition, $F(1,28)=57.84, p$ $<.001$. There was no significant main effect for the span group, $F(1,28)=0.83, p>.05$, and no significant inter-
Azuma, M., Ikeda, T., Minamoto, T., Osaka, M., \& Osaka, N. (2012) Working memory and eye movement control under Reading Span Test

action between span group and reading condition, $F$ (1, 28) $=3.37, p>.05$.

戦争に関する彼の 記憶は曖昧な点が多くてはっきりしない。

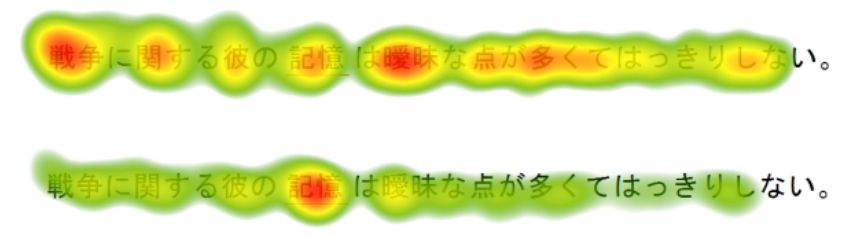

Figure 5. Example sentence of the two-sentence condition. Top panel: original Japanese sentence (English translation; Much of his memory regarding the war is unclear, underlined word is memory). Middle panel: The heatmap of 15 participants under the READ condition. . Bottom panel: The heatmap of the other 15 participants under the RST condition. The red color indicates the area much gazed.

The top portion of Figure 5 shows an original Japanese sentence and the red underline indicates the target word. The middle and lower section of Figure 5 depict sample heat maps for the two-sentence condition in the READ and the RST condition, respectively. These maps show that participants spent longer time on a target word when they needed to remember the word, while participants fixated on a target word as much as other word in the READ condition.

俳句では言葉の奥に込められた心情 を理解することが大切だ。

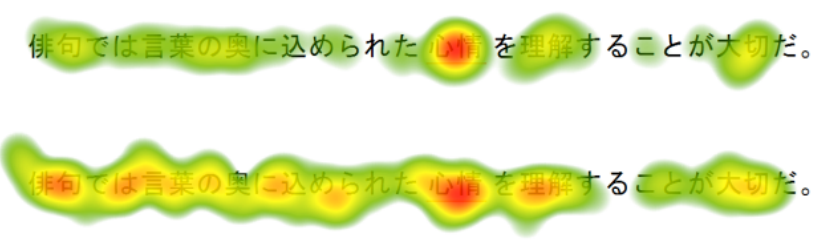

Figure 6. Example sentences under the two-sentence condition. Top panel: original Japanese sentence (English translation; It is critical to understand the sentiment involved within a word in Haiku, underlined word is sentiment). Middle panel: The heatmap of 7 high-span participants. Bottom panel: The heatmap of 8 low-span participants.

The top portion of Figure 6 shows similar panels of two-sentence condition. The middle and lower sections of Figure 5 depict sample heat maps for the RST condition in low-span and high-span participants, respectively. 
Journal of Eye Movement Research

$5(3): 1,1-10$

According to this figure, it seems that both HS and LS fixated on TBR words longer than other words.

\section{Five-sentence condition}

A $2 \times 2$ ANOVA with span group (HS and LS) and reading condition (RST and READ condition) was conducted. There was a main effect of the reading condition, $F(1,28)=47.57, p<.001$, but no main effect of the span group, $F(1,28)=1.32, p>.05$. There was a significant interaction between span group and reading condition in the five-sentence condition, $F(1,28)=10.71, p<.01$. Fisher's LSD analysis showed that the fixation time of the HS significantly longer than that of LS in the RST condition $(p<.05$.), whereas such group difference was not observed in the READ condition $(p>.05)$.

In addition, we computed the increase of fixation time by subtracting the duration in the READ condition from the RST condition and comparing the increase between HS and LS using a between-subject t-test. Results showed that, in the five-sentence condition, high-span participants showed a significantly greater increase in fixation time from the READ condition to the RST condition in comparison to the $\operatorname{LS}, t(1,28)=3.27, p<.01$. Such a difference was not observed in the two-sentence condition, $t(1,28)=1.84, p>.05$.

Although we found a significant interaction between span group and reading condition in the 5-sentences condition, but not in the 2-sentences condition, the result has to be interpreted carefully as the three-way interaction did not reach to the significant level $(p=.13)$.

\section{生徒たちは毎日重たい辞書を持って学校に通っている。}

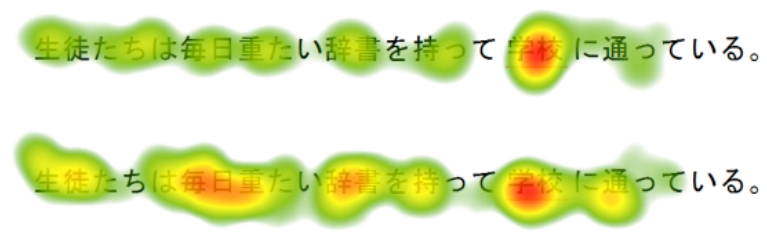

Figure 7. Example sentence of the 4th sentence under the fivesentence condition. Top panel: original Japanese sentence (English translation: The students go to school with heavy dictionary every day, underlined word is dictionary). Middle panel: The heatmap of 7 high-span participants. Bottom panel: The heatmap of 8 low-span participants.
Azuma, M., Ikeda, T., Minamoto, T., Osaka, M., \& Osaka, N. (2012 Working memory and eye movement control under Reading Span Test

Figure 7 displays sample heat maps while participants read a 4 th sentence in the five-sentence condition. The top panel shows an original Japanese sentence. The middle panel shows the distribution of fixation time of the low-span participants, and the bottom panel shows that of the high-span participants. In comparison to the twosentence condition, the five-sentence condition seems to be associated with highly distributed fixation points across the sentence in the LS, while fixation points in HS appear to be primarily placed on the target word. The scan paths of HS may indicate that they could efficiently avoid unnecessary fixations during reading and could control their eye movements precisely to the target words to be attended.

\section{Discussion}

The present study examined whether individual differences in working memory capacity are reflected in terms of the difference in eye movement while participants performed the Reading Span Test. We found a significant interaction between span group and memory load only in the more difficult condition (i.e., the five-sentence condition). Findings revealed that high-span participants tended to look at target words longer than low-span participants. Relative to the low-span group, high-span participants showed a larger increment in fixation time from the READ condition to the RST condition. This result suggests that participants spared more attentional resources in order to focus on the to-be-remembered words in the RST condition relative to the READ condition. This evidence suggests that individuals with high working memory spans efficiently control their eye movements in accordance with task demands. Thus, working memory capacity modulates eye movement under conditions of high memory load

The present results about total fixation time are consistent with the findings of Engle et al. (1992) who found that fixation time differed between high- and low- span participants. However, Engle et al. (1992) only tested a set size of 6 as high memory load, whereas the current study included high and low working memory conditions. Our results also support the results of Carpenter \& Just (1989), but we must consider the difference in procedure. On the other hand, our results do not support Kaakinen \& 
Hyönä's (2007) finding that participants with any working memory capacity showed a similar pattern of eye movements. As discussed previously, the prediction of a target location could remove the effect of working memory capacity on eye movement patterns. The present results support this view by demonstrating that high-span participants showed a longer fixation time in the more difficult condition in comparison to low span participants when the location of a target word was unpredictable. This group difference may reflect differences in the control efficiency of voluntary attention, such that high span participants may have spared more attentional resources to focus on target items while ignoring irrelevant words in sentences. The finding is in line with the previous literature showing that working memory capacity reflects general attentional control (Conway et al., 1999; Kane and Engle, 2003; Kane et al., 2004; Kane et al., 2007).

In contrast, low span participants may have their attention captured by non-target words in a sentence. As compared with the easy condition (i.e., the two-sentence condition), the difficult condition shows highly distributed fixation points across sentences in the low-span group, whereas the fixation point in the high-span group was mostly placed on the target word. The differing scan paths of high working memory performers may indicate that they could efficiently avoid unnecessary fixations during reading and control their eye movements precisely to the target words to be attended. We found similar results in functional magnetic resonance imaging (fMRI) studies using the RST for focused- and non-focused words (Osaka, Komori, Morishita \& Osaka, 2007). In the focused-RST (F-RST), the target word to be maintained was the focus word (i.e., critical word) in the sentence, whereas in the non-focused RST (NF-RST), the target word was not the focus word (i.e., less critical word therefore usually to-be- inhibited) in the sentence. Focusing attention is critical during span tasks. During the RST, at least two different functions are concurrently executed, namely, reading the sentences and memorizing the target words. While reading a sentence, the reader most likely searches for the most important word (i.e., the focus word), which plays a crucial role during the integration of a sentence (Osaka, Nishizaki, Komori, \& Osaka, 2002). Focusing attention also facilitates sentence comprehension (Carpenter \& Just, 1977). However, in the RST, the goal of the task is to remember specified target words while reading sentences, so that subjects are unable to maintain attention on the focus word of the sentence. In both RST conditions, we found significant brain activations in three main regions: the left dorsolateral prefrontal cortex (DLPFC), anterior cingulate cortex (ACC), and the left superior parietal lobule (SPL). In addition, fMRI signal changes increased in the left SPL for the NF-RST condition. Using fMRI, it appears that the neural substrates of focusing attention to the target word are based on SPL and ACC-DLPFC attentional networks. Furthermore, we found group differences in the focusing effect between high- and low-RST span participants. High span participants showed focusing effects greater than those of low span participants, which suggested greater ability to ignoring irrelevant information for high participants. The effect of syntactic focus is also examined with an eye movement approach. Birch and Rayner (1997) reported that focused word was spent more time than non-focusedword. To-be-remembered words in Japanese version of RST include both focused- and non-focused-word, whereas in the standard English RST such as Daneman and Carpenter (1980) version, most of to-be-remembered words are focused-words, since sentence-final word often have new information in English. Not only the position of to-be-remembered word but also this difference may contribute to difficulty of control attention in Japanese RST. Osaka, Nishizaki, Komori, \& Osaka, (2002) reported showed that low span participants reported a greater number of non-target word in a given sentence (intrusion error) in relative to high spans. It suggests that low-span group failed to inhibit the word with syntactic enhancement. Relating to the low-span groups' problem of inhibition, Vogel, McCollough, \& Machizawa (2005) reported that individuals with low capacity failed not to attend the task-relevant information into memory, but to inhibit the task-irrelevant information. Considering those finding into consideration, individuals with low span in our study might know where to allocate their attention, but fail to keep their attention on target word from other words. In fact, individuals with low-span also increased fixation time on target word under RST condition relative to READ condition, but not much as individuals with highspan, especially under the difficult condition, where the to-be-inhibited words increase.

As mentioned above, among the factors influencing RST score, an ability for inhibiting irrelevant information has been pointed out as one of the most critical factors. For example, Gernsbacher (1990) suggested that poor readers lack a good inhibitory mechanism and that this deficit is implicated in comprehension difficulty. In an 
experiment by Gernsbacher and Faust (1991), subjects were told to focus on either a picture or a word in the context display and to ignore the other. They were then asked to indicate whether a word in a test display was related to a word in the context display. The researchers found differences between skilled and less skilled readers in terms of ignoring an irrelevant stimulus. The suppression mechanism of less skilled readers is less efficient in suppressing the non-focused ignored information relative to more skilled readers. Therefore, performing a dual task, such as measuring the target word of each sentence, strongly requires the efficient suppression of an irrelevant word. Although high-level reading processes modulate eye movement programming by adjusting saccade size (Feng, 2009). Our current results showed fixation time may have influence as much as saccade size. Since eye movements play a critical role in reading, a number of studies have investigated the influence of mechanisms underlying the global effect during reading.

As for the whole sentence reading time, we found main effects of the reading condition and the task difficulty, but not the working memory capacity. The effect of the reading condition indicates that attentional resource for reading was less available under the dual task condition, slowing the reading time. Task difficulty also affected reading time, raising a possibility that large number of sentences might consume attentional resource. On the other hand, the critical factor, working memory capacity, did not affect the reading time and interact with other factors. This result is inconsistent with the previous studies (Carpenter and Just, 1989; Friedman and Miyake, 2004). Several methodological issues can be related to the inconsistent results, and one of them is the location of the target word. As already mentioned, to-be-remembered words were randomly assigned in a sentence in Japanese version of the RST while they were always placed at the end of a sentence in English version. In the former situation, both high and low working memory capacity groups may carefully read a sentence while searching a target word. Further studies are required to resolve the discrepancy between the previous studies and the present study.

There are several limitations in the present study. The first one is that we did not find the three-way interaction across factors (working memory capacity, reading condition and task difficulty). Although we found the significant two-way interaction between the working memory capacity and the reading condition in the 5-sentence con- dition but not in the 2-sentence condition, the differential effect of the task difficulty has to be cautiously interpreted. Another limitation is that we did not specify what kind of eye movement contributed to the increase in total fixation time in the high span group under higher memory load. Future studies need to investigate the issue with a sophisticated analysis.

\section{Conclusions}

The present study of eye movement control during reading showed that high working memory performers can efficiently control their eye movement in order to accomplish required task, and the individual differences between high and low working memory performers appears especially under high load memory task.

\section{References}

Baddeley, A. D. (1986). Working memory. New York: Oxford University Press.

Birch, S., \& Rayner, K. (1997), Linguistic focus affects eye movents during reading, Memory \& Cognition, 25,653-660.

Carpenter, P. A., \& Just, M. A. (1989). The role of working memory in language comprehension. In D. Klahr \& K. Kotovsky (Eds.), Complex information processing: The impact of Herbert A. Simon (pp. 31-68). Hillsdale, NJ: Lawrence Erlbaum Associates Inc.

Conway, A. R. A., Tuholski, S. W., Shisler, R. J., \& Engle, R. W. (1999). The effect of memory load on negative priming: An individual differences investigation. Memory \& Cognition, 27, 1042-1050.

Daneman, M. \&Merikle, P. M. (1996). Working memory and language comprehension: A meta-analysis. Psychonomic Bulletin \& Review, 3, 422-433. 
Journal of Eye Movement Research

$5(3): 1,1-10$

Engle, R. W., Cantor, J., \&Carullo, J. J. (1992). Individual differences in working memory and comprehension: A test of four hypotheses. Journal of Experimental Psychology: Learning, Memory, and Cognition, 18, 972-992

Feng, G. (2009). Time course and hazard function: A distributional analysis of fixation duration in reading. Journal of Eye Movement Research,3, 1-23.

Friedman, N. P., \& Miyake, A. (2004). The reading span test and its predictive power for reading comprehensiom ability. Journal of Memory and Language, 51,136-158.

Gernsbacher, M. A., (1990). Less skilled readers have less efficient suppression mechanisms. Psychological Science, 4, 294-298.

Gernsbacher, M. A., \& Faust, M. E., (1991). The mechanism of suppression: A component of general comprehension skill. Journal of Experimental Psychology: Learning, Memory, \& Cognition, 17, 245-262.

Just, M. A. \& Carpenter, P. A. (1992). A capacity theory of comprehension: Individual differences in working memory. Psychological Review, 99, 122-149.

Kaakinen, J. K. \&Hyönä, J. (2007). Strategy use in the reading span test: An analysis of eye movements and reported encoding strategies. Memory, 15, 634-646.

Kane, M..J., Conway, A..R.A.., Hambrick, D. Z., \& Engle, R. W. (2007). Variation in working memory capacity as variation in executive attention and control. In A.R.A. Conway, C.Jarrold, M.J. Kane, A. Miyake, and J.N. Towse (Eds.), Variation in Working Memory (pp. 21 - 48). NY: Oxford University Press.

Kane, M. J., \& Engle, R. W. (2003). Working-Memory Capacity and the Control of Attention: The Contributions of Goal Neglect, Response Competition and Task Set to Stroop Interference, Journal of Experimental Psychology: General, 132, 47-70.

Kane, M. J., Hambrick, D. Z., Tuholski, S. W., Wilhelm, O., Rayne, T. W., \& Engle, R. W. (2004). The Generality of Working Memory Capacity: A LatentVariable Approach to Verbal and Visuospatial Memory Span and Reasoning. Journal of Experimental Psychology: Genral, 133, 189-217.
Azuma, M., Ikeda, T., Minamoto, T., Osaka, M., \& Osaka, N. (2012)

Working memory and eye movement control under Reading Span Test

Osaka, M., \& Osaka, N. (1992). Language-independent working memory as measured by Japanese and English reading span tests. Bulletin of the Psychonomic Society, 30, 287-289.

Osaka, M., Osaka, N., \& Groner, R. (1993). Languageindependent working memory: Evidence from German and French reading span tests. Bulletin of the Psychonomic Society, 31, 117-118.

Osaka, M., Nishizaki, Y., Komori, M., \& Osaka, N. (2002). Effect of focus on verbal working memory: critical role of the focus word in reading. Memory \&Cognition, 30, 562-571.

Osaka, M. \& Osaka, N. (1994). Working memory capacity related to reading: Measurement with the Japanese version of reading span test. Japanese Journal of Psychology, 65, 339-345.

Osaka, M., Komori, M., Morishita, M., \& Osaka, N., (2007). Neural bases of focusing attention in working memory: An fMRI study based on group differences. Cognitive, Affective, Behavioral Neuroscience, 7 , 130-139.

Osaka, N. (1994). Size of saccade and fixation duration of eye movements during reading: psychophysics of Japanese text processing. Journal of the Optical Society of America, $A$ 9, 5-13.

Rayner, K. (2008). Eye movements in reading: models and data. Journal of Eye Movement Research, 2, 1-10.

Vitu, F. (2008). About the global effect and the critical role of retinal eccentricity: Implications for eye movements in reading. Journal of Eye Movement Research, 2, 1-18.

Vogel. E. K., McCollough, A. W., \& Machizawa, M. G. (2005), Neural measures reveal individual differences in controlling access to working memory. Nature, 438. 500-503.

\section{Acknowledgements}

This work was supported by grants from the Japan Society for the Promotion of Science (\#22220003) to N.O., and in part by Global COE Program "Center of HumanFriendly Robotics Based on Cognitive Neuroscience" of the Ministry of Education, Culture, Sports, Science and Technology, Japan. 